\title{
Flexible Parylene Packaged Intraocular Coil for Retinal Prostheses
}

\author{
Wen $\mathrm{Li}^{1}$, Damien C. Rodger ${ }^{1,2}$, Ellis Meng ${ }^{3}$, James D. Weiland ${ }^{2}$, Mark S. Humayun ${ }^{2}$, and Yu-Chong Tai ${ }^{1}$ \\ ${ }^{1}$ California Institute of Technology \\ 1200 E. California Blvd. M/C 136-93, Pasadena, CA 91125, USA \\ Tel: +1-626-395-3885, Fax: +1-626-584-9104, E-mail: wenli@mems.caltech.edu \\ ${ }^{2}$ Keck School of Medicine, University of Southern California \\ Health Science Campus, University of Southern California, Los Angeles, CA 90033, U.S.A. \\ ${ }^{3}$ University of Southern California \\ University Park Campus, University of Southern California, Los Angeles, CA 90089, U.S.A.
}

\begin{abstract}
We present a flexible and fully-implantable coil designed for use as a power and data transfer component in retinal prosthesis applications. Compared with traditional hand-made intraocular coils, this microfabricated coil is flexible, with a $9.5 \mathrm{~mm}$ outer diameter and $10-\mu \mathrm{m}$-thick parylene $\mathrm{C}$ as the primary structural and packaging material. A post-fabrication heat treatment was used to improve the parylene package in order to protect the device in harsh corrosive environments such as the human eye. Long-term accelerated-lifetime soak testing in heated saline has been performed, and the mean time to failure (MTTF) of the parylene package extrapolated to $37^{\circ} \mathrm{C}$ was estimated using the Arrhenius relationship. The electrical failure of this device was also characterized by measuring the DC resistance in saline.
\end{abstract}

Keywords: Accelerated-lifetime soak testing, Coil, Intraocular, Parylene, Retinal prostheses

\section{INTRODUCTION}

Inductive-coupling of a pair of coils is a promising technology for wireless power and data transmission to the implanted components of many biomedical devices, and in particular for retinal prostheses [1]. In such an implementation, the secondary coil, which would reside inside the human eye, has to be mechanically durable, flexible, and small enough for intraocular implantation. It should also be chemically stable in order to prevent harmful interaction with the surrounding tissues. The current state-of-the-art coil for intraocular retinal prostheses is a hand-wound coil [2], which is thick and very stiff, and can cause notable degradation in the region of implantation. Therefore, we propose a new parylene-based thin-film technology incorporating multi-layer metal conductive wires to accomplish the function of this intraocular implanted device. Figure 1 gives an overall depiction of the intraocular coil design. In this design, at least two layers of metal are required in order to enable a closed circuit with two perimeter contacts. The two layers of metal are interconnected via the through-hole in the parylene insulating layer, and are completely encapsulated by parylene C.

Parylene $\mathrm{C}$ is ideal as the primary structural and packaging material due to its desired properties, including flexibility (elongation to break $\sim 200 \%$ ) and mechanical strength (Young's modulus $\sim 4 \mathrm{GPa}$ ), chemical inertness, United States Pharmacopeia (USP) Class VI biocompatibility [3], and low water permeability when compared with other materials, such as polyimide [4]. Moreover, parylene $\mathrm{C}$ is a room temperature CVD-coated material. Thus the packaging method does not require bonding, high temperatures, or large electric fields which may potentially damage circuitry. The device described herein is fully microfabricated in a manner compatible with multielectrode arrays [5], and parylene-based packages incorporating application specific integrated circuits (ASICs), or other parylene-packaged discrete electrical components [3].

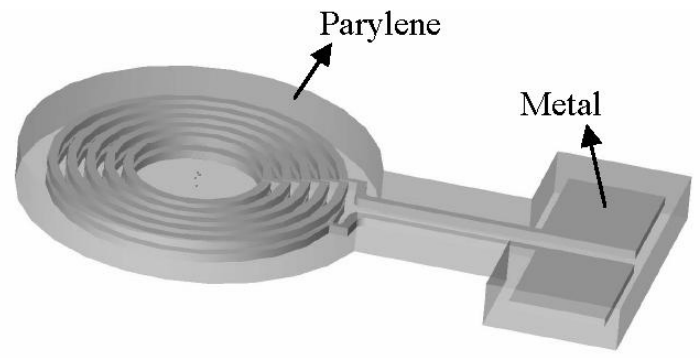

Figure 1. Illustration of the intraocular coil concept.

\section{DEVICE FABRICATION}

The intraocular coil is fabricated using a parylene/metal thin-film technique, as illustrated in Figure 2. Briefly, a layer of photoresist is first spun on a standard silicon wafer as a sacrificial layer, and a $5 \mu \mathrm{m}$ layer of parylene is vapor-deposited on the substrate. Then, a $550 \mathrm{~nm}$ 
multi-layer metal of $\mathrm{Ti} / \mathrm{Au} / \mathrm{Ti}$ is deposited using e-beam evaporation. After metal wet etching, a $2 \mu \mathrm{m}$ layer of parylene is deposited as an insulating layer, and is patterned using oxygen plasma reactive-ion etching to create the interconnection via between the two layers of metal. The second layer of metal is then deposited using optimized step coverage and patterned. After that, another parylene layer is deposited so that the metal wires are entirely encapsulated. A photoresist mask is then patterned to define the coil profile, followed by parylene etching using oxygen plasma to open the contact pads and define the overall coil geometry. Finally, the entire flexible structure is released from the silicon substrate by dissolving the photoresist sacrificial layer using a standard acetone etch process. Although this technology is specifically developed for the intraocular coil fabrication, it can be used for making other flexible devices with multi-layer metals as well, such as multielectrode arrays.

1.Spin sacrifacial photoresist. Deposit first layer parylene

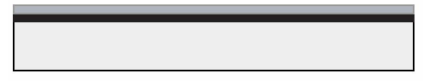

2. Deposit first layer metal. Spin, expose and develop photoresist. Etch metal

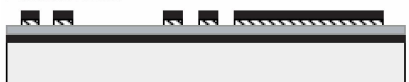

3.Deposit second layer parylene. Spin, expose and develop photoresist. Transfer pattern with $\mathrm{O}_{2}$ plasma

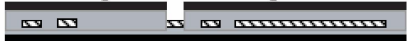

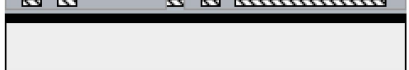

\section{Strip
thin film}

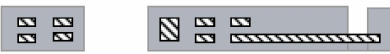

\begin{tabular}{|llll|}
\hline Q Metal & Photoresist & Parylene & $\square$ Silicon \\
\hline
\end{tabular}

Figure 2. Process flow for intraocular coil fabrication.

A fabricated intraocular coil is shown in Figure 3(a), and Figure 3(b) shows the interconnection via between the two layers of metal. This coil prototype has 10 turns of metal wires with an outer diameter of $9.5 \mathrm{~mm}$, and a total thickness less than $11 \mu \mathrm{m}$. Compared with the conventional hand-wound coil, this parylene-based coil is very flexible and foldable because of the unique properties of parylene $\mathrm{C}$. Figure 4 demonstrates the flexibility of this $10-\mu \mathrm{m}$-thick parylene /metal thin-film skin.

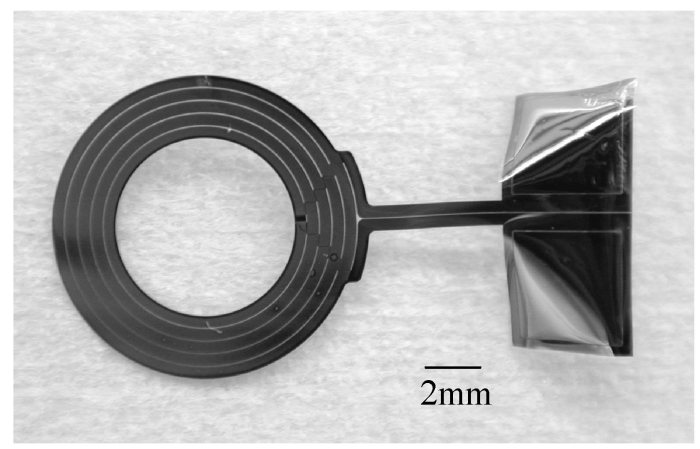

(a)

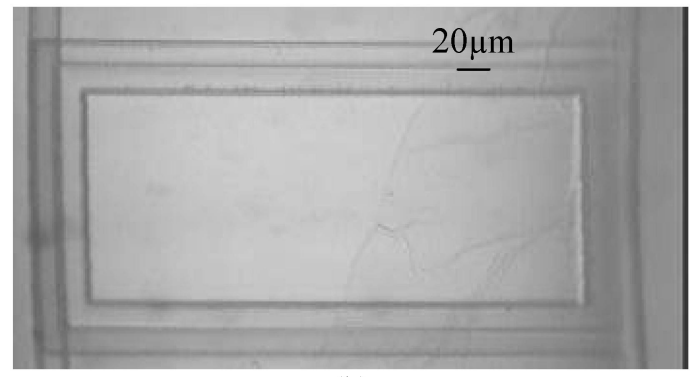

(b)

Figure 3. (a) A fabricated intraocular coil; (b) The interconnection via between the two metal layers.

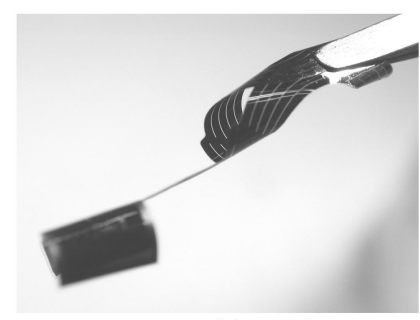

(b)

Figure 4. Flexibility of the parylene/metal thin-film skin: (a) skin folded in half; (b) skin rolled into a tube.

\section{RESULTS AND DISCUSSIONS}

The AC resistances and the self-inductances of the intraocular coil measured with an HP 4192A are depicted in Figure 5. At the target frequency of $1 \mathrm{MHz}$ for the envisioned retinal prosthesis system, the self-inductance is approximately $0.7 \mu \mathrm{H}$, and the series resistance is approximately $13 \Omega$, as expected from our design. 


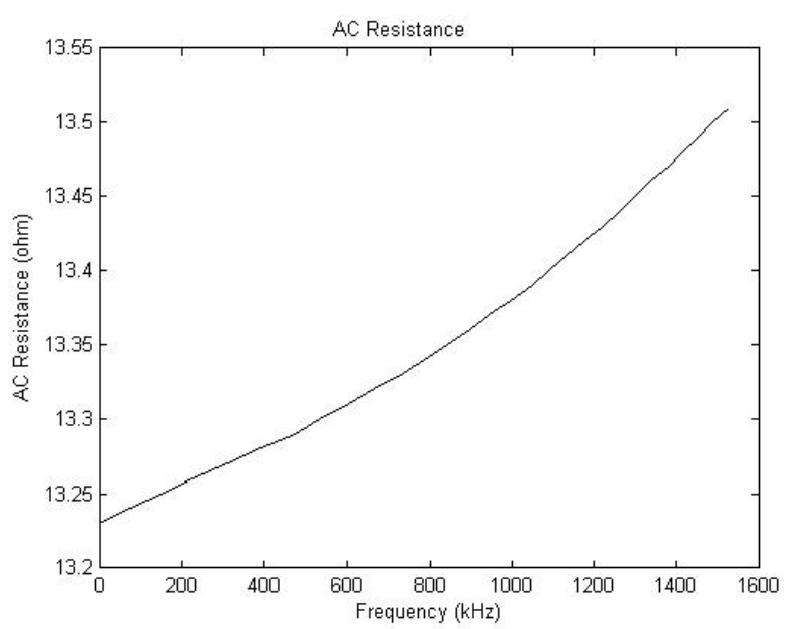

(a)

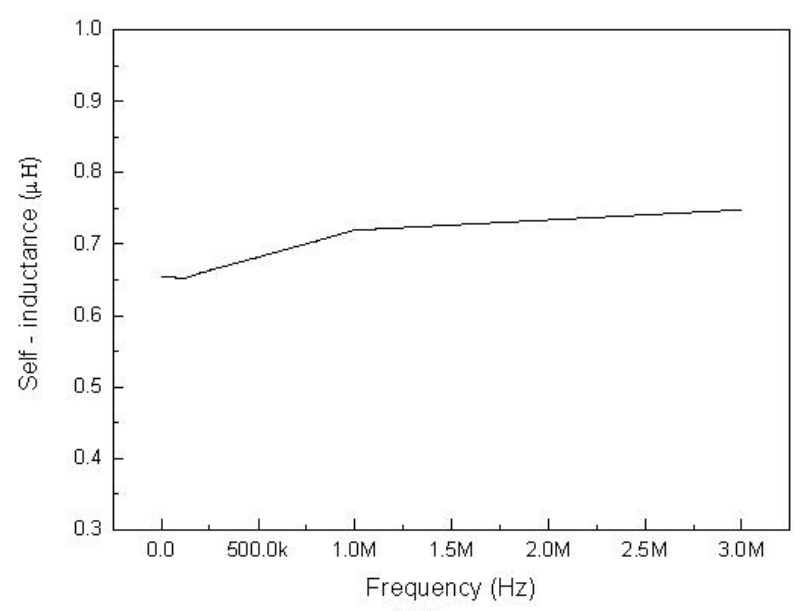

(b)

Figure 5. (a) Measured $\mathrm{AC}$ resistance of the intraocular coil; (b) Self-inductance vs. frequency.

Electromagnetic coupling has been demonstrated using a simplified inductive data link with a pair of coils, in which the primary coil is a hand-wound coil with a self-inductance of $23 \mu \mathrm{H}$, and the secondary coil is our microfabricated intraocular coil. The two coils were separated by a distance of $11 \mathrm{~mm}$, and the primary coil was driven by a standard function generator with a sinusoidal input signal of $20 \mathrm{~V}$ peak to peak. Both the primary and secondary circuits were tuned at the same resonance frequency of $1 \mathrm{MHz}$. Under measurement, a received signal of $120 \mathrm{mV}$ peak to peak was seen on the secondary coil, which indicates successful signal transmission, as plotted in Figure 6. However, only a small amount of transferred power was detected using this intraocular coil. This is mainly because of the low inductance and small $\mathrm{Q}$ factor $(\sim 0.3)$ of the coil. For future versions of intraocular coils, it is possible to improve the performance by varying the device design in terms of width, metal thickness, or number of layers.

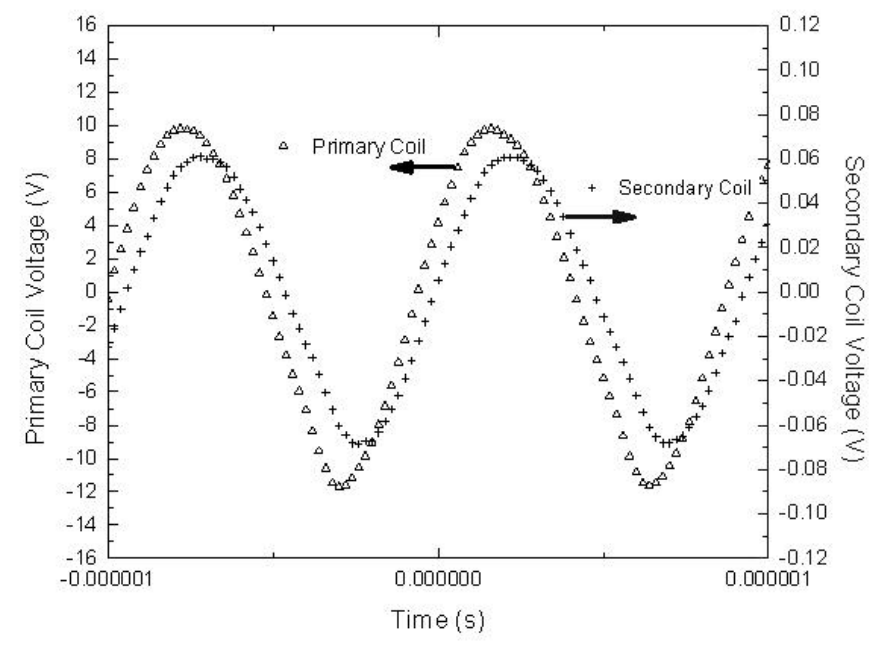

Figure 6. Demonstration of coupling effect using the intraocular coil.

In order to evaluate the performance of the parylene $\mathrm{C}$ package, long-term accelerated-lifetime soak testing was performed in saline at a higher temperature than the final-use (body) temperature for retinal prosthesis applications. The soak test samples were prepared using the parylene/metal thin-film technology described here. A post-fabrication heat-treatment was performed in a vacuum oven with $\mathrm{N}_{2}$ backfill at $200^{\circ} \mathrm{C}$. After that, the devices were immersed in saline either at $90{ }^{\circ} \mathrm{C}$ or $77^{\circ} \mathrm{C}$. The samples were then observed under optical analysis at one day intervals to monitor for device failure. Bubbles and delamination were seen in the sample soaked at $90^{\circ} \mathrm{C}$ after 23 days, while no delamination or major physical corrosion occurred in the other sample immersed at $77{ }^{\circ} \mathrm{C}$ for over 80 days. Continuous soak testing at $77{ }^{\circ} \mathrm{C}$ is still underway. Assuming an Arrhenius relationship exists, it can be predicted that the parylene package can remain in intact at the body's normal temperature of $37^{\circ} \mathrm{C}$ for over 20 years [6].

To characterize the electrical failure of the devices, active accelerated-lifetime soak testing was also performed by immersing the coil into saline at $75^{\circ} \mathrm{C}$ with a fixed through current of $16 \mathrm{~mA}$, simulating the expected instantaneous stimulation and chip-operation current in the final implementation of the system. The dynamic response of coil DC resistance was monitored with an HP 4145B at various time intervals, as shown in Figure 7. After 28 days under these accelerated-lifetime conditions, the DC resistance dramatically rose to $21.9 \mathrm{k} \Omega$, indicating that failure occurred. This preliminary result shows that the lifetime of the intraocular coil will likely be shorter when there is a current applied through the device. More soak tests need to be performed in order to fully understand the failure mode of the parylene/metal thin-film skin. Further improvements can be achieved by optimizing the temperature and duration of heat treatment for the parylene/metal thin-film skin or by using additional chemical treatments. 


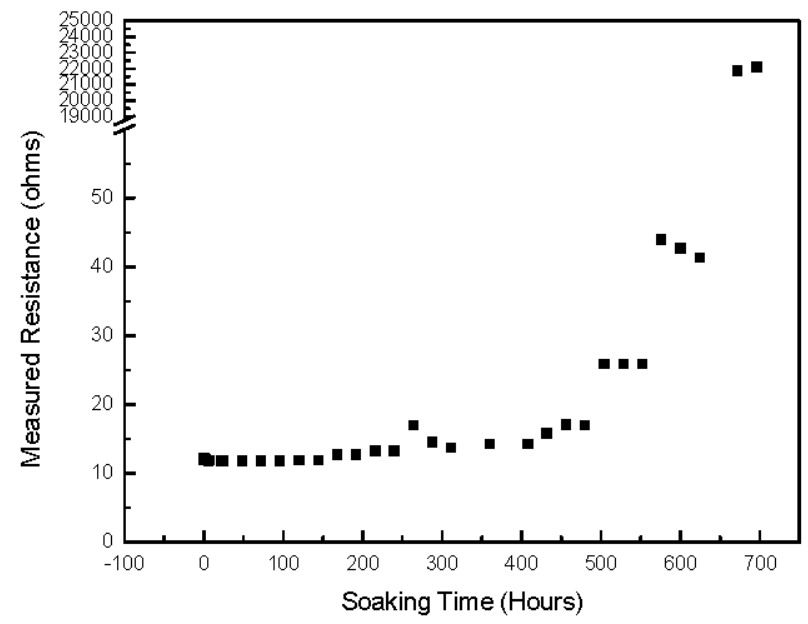

Figure 7. Dynamic response of the resistance when the intraocular coil was immersed in saline at $75^{\circ} \mathrm{C}$

\section{CONCLUSIONS}

We have designed and successfully fabricated a flexible intraocular coil for retinal prostheses using the parylene/metal skin microfabrication technique. Because of the unique properties of parylene, this device is flexible and foldable so as to enable implantation into the eye through a small surgical incision. Preliminary test results demonstrate that a signal can be successfully transmitted using an inductive data link. To improve the parylene package, a post-fabrication heat treatment has been developed. Long-term accelerated-lifetime soak testing suggests a mean time to failure at body temperature of over 20 years, which demonstrates the near-hermetic biocompatible package using parylene $\mathrm{C}$ for implanted devices. However, these results are still preliminary, and need to be extended in the future in order to more fully study the failure mechanism of the parylene package.

\section{ACKNOWLEDGMENTS}

This work was supported in part by the Engineering Research Centers Program of the National Science Foundation under Award Number EEC-0310723 and by a fellowship from the Whitaker Foundation (D.R.). The authors would also like to thank Mr. Trevor Roper and other members of the Caltech Micromachining Laboratory for their assistance with fabrication.

\section{REFERENCES}

[1] M. S. Humayun, "Intraocular retinal prosthesis," Tr. Am Ophth Soc, vol. 99, pp. 271-300, 2001.

[2] T. Stieglitz, W. Haberer, C. Lau, and M. Goertz, "Development of an inductively coupled epiretinal vision prosthesis," in Proc. IEEE-EMBS, San Francisco, CA, USA, Sept. 1-5, 2004, pp. 4178-4181.

[3] D. C. Rodger, J. D. Weiland, M. S. Humayun, and Y. C. Tai, "Scalable flexible chip-level parylene package for high lead count retinal prostheses," in Proc. Transducers, Seoul, South Korea, June 5-9, 2005, pp.1973-1976.

[4] J. J. Licari and L. A. Hughes, Handbook of polymer coating for electronics: chemistry, technology, and applications, $2^{\text {nd }}$ ed. Park Ridge, N. J., U.S.A.: Noyes Publications, 1990.

[5] D. C. Rodger, W. Li, H. Ameri, A. Ray, J. D. Weiland, M. S. H., and Y. C. Tai, "Flexible parylene-based microelectrode technology for intraocular retinal prostheses," in Proc. IEEE-NEMS, Zhuhai, China, Jan. 18-21, 2006.

[6] Brian H. Stark, Khalil Najafi, "An ultra-thin hermetic package utilizing electroplated gold," in Proc. Transducers, Munich, Germany, June 2001. 